American Journal of Applied Sciences 6 (3): 414-423, 2009

ISSN 1546-9239

(C) 2009 Science Publications

\title{
Georadar Measurements for the Snow Cover Density
}

\author{
A. Godio \\ DITAG, Politecnico di Torino, C.So Duca degli Abruzzi, 24-I-10129 Torino, Italy
}

\begin{abstract}
Ground Probing Radar (GPR) devices is adopted for the analysis of thickness and the mechanical properties (density) of the snow cover in some test site in Alps, in Northern Italy. The performances of standard radar systems for the snow cover characterisation are analysed, the main aim is to assess the reliability of the method to estimate the snow density, the snowpack thickness and the depth resolution in terms of capability to detect thin layers. The main relationships between the electrical permittivity and the density of the dry-snow are applied to estimate the density vertical profiles inferred by the GPR investigation. The data were calibrated and compared with the results coming from direct measurements of the density and thickness.
\end{abstract}

Key words: Applied electromagnetism, snow density, georadar

\section{INTRODUCTION}

The estimation of snow density and the water equivalent are significant in snow-engineering because the snow water equivalent determines the amount of snow-melt discharge that is a factor in runoff modelling and hence in designing a drainage system with economic impact if the snow-melt-water is to be used for power generation. Moreover as snow-melt water carries pollution from snow dumps, snow density and water equivalent estimation are parameters related to the contaminant transport modelling. The measurement of the vertical density profiles assumes great interest being density a mechanical properties that can be related to the avalanche risk in mountainous areas ${ }^{[1]}$, furthermore different degrees of snow metamorphism can be recognised by density estimation, spatial changes and time-varying of density along vertical profiles must be considered for estimating the avalanche risk.

Ground-based surveys using portable electromagnetic equipment be adopted for a fast and extensive estimate of thickness and average density of the snow pack as reported in several papers ${ }^{[2,3]}$. The combined use of high (using TDR-Time Domain Reflectometry) and low-frequency permittivity measurements is usually performed for continuous snow wetness and snow density determination ${ }^{[4]}$, new electromagnetic sensors for large-scale snow cover monitoring are continuously developing ${ }^{[5]}$. The observed electromagnetic parameters are related to the density values of the dry or wet snow using empirical rule or mixing models. The reliability of high frequency radar devices (around $10 \mathrm{GHz}$ ), particularly stepped- frequency systems, to relate the mechanical properties of thin layers with the georadar response has been demonstrated ${ }^{[6]}$.Otherwise the application of these systems on wide areas is not yet well documented. On the other hand impulse radar systems offer a robust tool for mapping wide areas at low cost to estimate density and thickness of the snow cover.

In such a context the paper deals with a short description of the relationships between the electrical permittivity and the snow density. The results of experiments of ground probing radar survey conducted in several sites in the Italian Alps, characterised by different snow conditions are interpreted in terms of density vertical profiles. The results of the georadar interpretation were calibrated and compared with the density estimate using conventional approach.

The experimental results here discussed show that impulse radar systems are capable to point out and assess the lateral continuity of interesting features in the snow pack, such as ice crusts, that are relevant in the avalanche risk estimation.

Snow density and electromagnetic models: The density forming processes can be classified as primary, which develops at the snow surface either during deposition or by subsequent reworking from wind and secondary, which evolves on site subsequent to the deposition due to thermo-mechanical processes.

The primary densification depends on the climate conditions, the precipitation rate, the size and type of snow crystals and the packing and disintegration of crystals by wind. The secondary densification results from volumetric creep due to normal and shear stresses and by the linked processes of heat and mass transport. 
The bulk density of the snow cover can be computed according to the contribute of each components:

$$
\rho_{\mathrm{m}}=\left[\rho_{\text {ice }} \cdot(1-\phi)+\rho_{\text {water }} \cdot \theta\right]
$$

where, $\phi$ is the snow porosity and $\theta$ is the free water content. The water content depends on the temperature and transformations of the snow pack. Fresh snow has a very low free water content (less than 3\%), while in spring during snow melting the water content can reach values above $15 \%$. An increase of the mass, for instance due to fresh snow precipitation, provides for a compaction of the layers with reduction of air volume or melt of old snow and transformation in ice or liquid water. The air temperature during the snowfalls influences the density of fresh snow, density ranges from $20 \mathrm{~kg} \mathrm{~m}^{-3}$ for temperature of-20 Celsius up to 200 $\mathrm{kg} \mathrm{m}^{-3}$ for temperature close to zero. The fresh snow density is related to other factors such as the shape of the snow crystals and the temperature gap between the crystal and ground. Finally the thickness and the compaction of the old snow can modify the thermal circulation between subsoil and water and could influence the metamorphism of snow pack.

The massive ice can be considered as an aggregate of single ice crystals and therefore it is similar to a mono-crystalline rock, the fresh snow or firm snow (granular snow) could be assimilated to a sedimentary rock and finally glacial ice is similar to a metamorphic rock.

The snow electrical conductivity depends on the porosity (inversely proportional to the bulk density), the morphology of the snow crystals, the metamorphism, the water content and temperature. For density ranges between $240 \mathrm{~kg} \mathrm{~m}^{-3}$ (fresh snow) to $640 \mathrm{~kg} \mathrm{~m}^{-3}$ (granular snow), Kopp ${ }^{[7]}$ showed that the electrical conductivity ranges between $10^{-9}-10^{-7} \mathrm{~S} \mathrm{~m}^{-1}$ at temperature of $-10^{\circ}$ Celsius. An increase of the conductivity can be observed at temperature close to $0^{\circ}$ Celsius where values of $10^{-5} \mathrm{~S} \mathrm{~m}^{-1}$ are recorded.

The electrical permittivity indicates the polarizability of a material: as the frequency increases the polarisation response may lag behind the varying field, therefore the general form of permittivity involves an in-phase $\left(\varepsilon^{\prime}\right)$ and out of phase ( $\left.\varepsilon^{\prime \prime}\right)$ component:

$$
\varepsilon=\varepsilon^{\prime}-\mathrm{i} \varepsilon^{\prime \prime}
$$

where, $\varepsilon$ " is also called the loss factor. The loss factor (tangent) of the snow depends on the percentage of the free liquid water content. A value for loss tangent for a specific snow may be inapplicable by several orders of magnitude for other snows with different amounts of liquid water.

The electrical permittivity of the snow can be estimated according to the assumption that snow is a mixture of air, water and ice, the electromagnetic properties of the dry compact snow (with water content below $3 \%$ ) are very similar to the ice dielectric value $\left(\varepsilon_{\text {ice }}=3,2\right)$.

From an electrical point of view the dry-snow can be considered as non-conducting medium, the electromagnetic wave does not suffer of the intrinsic attenuation as it propagates through the snowpack and it can be assimilated to a lossless medium, in such a case the complex permittvity $\varepsilon_{\mathrm{c}}$ is equal to the real permittivity $\varepsilon$ and the following relationships yield, the wavenumber $\mathrm{k}$ is defined by:

$$
\mathrm{k}=\omega \sqrt{\mu \cdot \varepsilon}
$$

and the phase-wave velocity is:

$$
\mathrm{v}=\frac{\mathrm{k}}{\omega}=\frac{1}{\sqrt{\mu \cdot \varepsilon}}
$$

where, $\mu$ is the magnetic permeability of the medium $\left(4 \pi 10^{-7}\right.$ Henry/m in the S.I. unit) and $\varepsilon$ is the dielectric constant of vacuum $\left(8.85210^{-12} \mathrm{~F} \mathrm{~m}^{-1}\right)$.

The intrinsic impedance is:

$$
\eta=\sqrt{\frac{\mu}{\varepsilon}}
$$

The phase velocity and the wavelength are related by:

$$
\lambda=\frac{2 \cdot \pi}{\mathrm{k}}=\frac{\mathrm{v}}{\mathrm{f}}
$$

the wavelength $(\lambda)$ of the travelling wave is proportional to the phase velocity and inversely to the frequency (f).

At the boundary between two snow layers or between the snowpack and the bedrock, for a normal plane wave incidence, the reflection $(\Gamma)$ and transmission coefficient $(\tau)$ are:

$$
\begin{gathered}
\Gamma=\frac{\eta_{2}-\eta_{1}}{\eta_{2}+\eta_{1}} \\
\tau=\frac{2 \eta_{2}}{\eta_{2}+\eta_{1}}
\end{gathered}
$$


and for non magnetic materials (as the snow):

$$
\Gamma=\frac{\sqrt{\varepsilon_{1}}-\sqrt{\varepsilon_{2}}}{\sqrt{\varepsilon_{1}}+\sqrt{\varepsilon_{2}}}
$$

The performance of the radar survey can be estimated a priori, considering the response of a single thin layer as a rough planar target radar response. In such a context the target is defined as a rough planar target when the ratio between the surface roughness and the wavelength is greater than $1 / 2 \pi$. The wavelength in the frequency range between $500 \mathrm{MHz}$ and $1000 \mathrm{MHz}$ is $0.1-0.2 \mathrm{~m}$. Therefore, it is appropriate to consider the thin snow layer as a rough planar target especially when high frequencies (>900 MHz) are adopted.

The radar cross section of the planar rough target refers to the First Fresnel Zone and it is defined by $\Gamma^{2} \pi \lambda \mathrm{D} / 2$, where $\mathrm{D}$ is the object-antenna distance (in the single reflection zero-offset mode) and $\Gamma^{2}$ is the plane wave power reflection coefficient. The maximum penetration depth can be determined taking into account to the performances, the sensibility of the radar system and the antenna gains. From a theoretical point of view at the main frequency of $900-1000 \mathrm{MHz}$, a good compromise between the penetration depth (more than $3 \mathrm{~m}$ ) and the vertical resolution could be obtained, the vertical resolution is the minimum vertical distance between two interfaces (e.g., snow layers with different density) that can be distinguished in the radar images as two well separated events. This depends on the radar performances and the wavelength of the signal and therefore it depends on the density of the snow pack. For instance a granular high density snow $\left(>600 \mathrm{~kg} \mathrm{~m}^{-3}\right.$ ) presents a wavelength of $0.2 \mathrm{~m}$ (at $1 \mathrm{GHz}$ ) and a theoretical vertical resolution of $0.05 \mathrm{~m}$ (1/4 of the wavelength).

For a thin layer of a medium 2 which is embedded into a medium 1, if the thickness $t$ of the second layer is comparable to the wavelength in the first medium, a more appropriate expression for the reflection coefficient is:

$$
\Gamma=\frac{\Gamma_{12} \cdot\left(1-\mathrm{e}^{\mathrm{i} \beta}\right)}{1-\Gamma_{12}^{2} \mathrm{e}^{\mathrm{i} \beta}}
$$

where, $\beta=\frac{4 \cdot \pi \cdot \mathrm{t}}{\lambda_{1}}$ with $\lambda_{1}$ the wavelength in medium 1 .

The Robin's equation ${ }^{[8]}$ is an empirical relationship between density and electrical permitivity:

$$
\varepsilon_{\mathrm{r}, \mathrm{ice}}=(1+0.845 \cdot \rho)^{2}
$$

A simple mixing model could be adopted for describing the macroscopically behaviour of the electrical permittivity of the mixture of ice, air and water ${ }^{[9]}$ :

$$
\varepsilon=\left[\varepsilon_{\text {ice }}^{0.5} \cdot(1-\phi)+\varepsilon_{\text {air }}^{0.5} \cdot(\phi-\theta)+\varepsilon_{\text {water }}^{0.5} \cdot \theta\right]^{2}
$$

where, the permittivity of the mixture $(\varepsilon)$ is the volume weighted average of the contribute of the permittivity of the snow crystals $\left(\varepsilon_{\text {ice }}\right)$, the water $\left(\varepsilon_{\text {water }}\right)$ and the air.

In this study the following relative values of the electrical permittivity are considered: $\varepsilon_{\text {ice }}=3.2$, $\varepsilon_{\text {water }}=78$ (at $0^{\circ}$ Celsius) and $\varepsilon_{\text {air }}=1$, the density of the snow crystals is the ice density equivalent to $920 \mathrm{~kg} \mathrm{~m}^{-3[10]}$. The dielectric behaviour of dry-snow with respect to the density can analysed according to the relationship formulated by Looyenga ${ }^{[11]}$.

$$
\varepsilon_{\mathrm{r}, \text { snow }}^{1 / 3}-1=\mathrm{x} \cdot\left(\varepsilon_{\mathrm{r}, \text { ice }}^{1 / 3}-1\right)
$$

where, $x=\frac{\rho_{\text {mixture }}}{\rho_{\text {ice }}}$ is the ratio between the density of snow mixture and ice $\left(920 \mathrm{~kg} \mathrm{~m}^{-3}\right)$.

The theoretical relations between the density and the electromagnetic parameters in the snowpack are estimated and plotted in Fig. 1. Table 1 shows some theoretical values of the electromagnetic parameters for different snows.
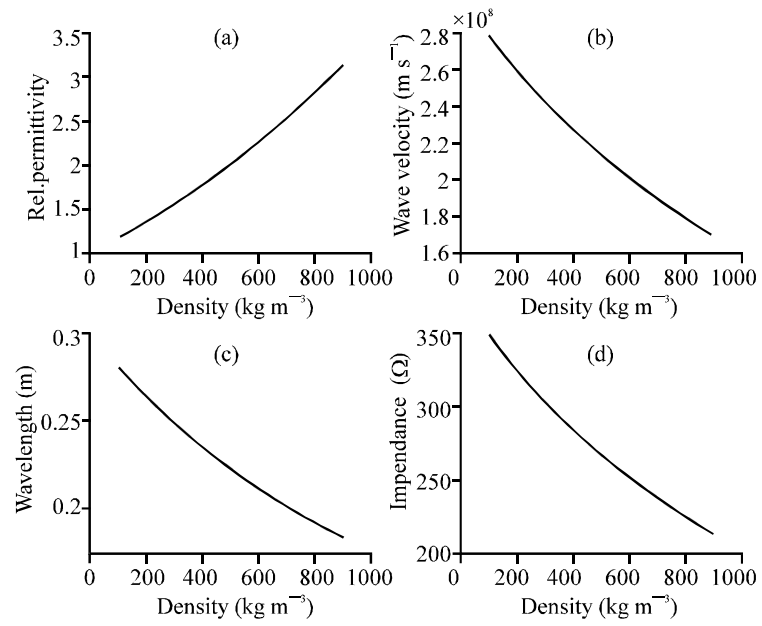

Fig. 1: Theoretical trend of the electrical permittivity of snow (a), wave velocity (b), wavelength (c) and electrical impedance (d), according to the Looyenga model 
Am. J. Applied Sci., 6 (3): 414-423, 2009

Table 1: Theoretical values of electromagnetic properties for different snow density (1 GHz)

\begin{tabular}{llllll}
\hline Snow & $\begin{array}{l}\text { Density range } \\
\left(\mathrm{kg} \mathrm{m}^{-3}\right)\end{array}$ & $\begin{array}{l}\text { Theorethical wave } \\
\text { velocity* }\left(\mathrm{m} \mathrm{sec}^{-1}\right) * 10^{8}\end{array}$ & $\begin{array}{l}\text { Electrical } \\
\text { Permittivity }\end{array}$ & $\begin{array}{l}\text { Wavelength } \\
(\mathrm{m})\end{array}$ & $\begin{array}{l}\text { Impedance } \\
(\text { ohm })\end{array}$ \\
\hline Fresh- snow & 100 & 2.78 & 1.16 & 0.28 & 349.4 \\
& 300 & 2.42 & 1.54 & 0.24 & 303.7 \\
Granular snow & 400 & 2.27 & 1.75 & 0.27 & 284.5 \\
& 600 & 2.00 & 2.24 & 0.20 & 251.6 \\
Ice crust & 700 & 1.89 & 2.52 & 0.19 & 237.4 \\
& 900 & 1.69 & 3.13 & 0.17 & 212.8 \\
\hline
\end{tabular}

*: According to the Eq. 8

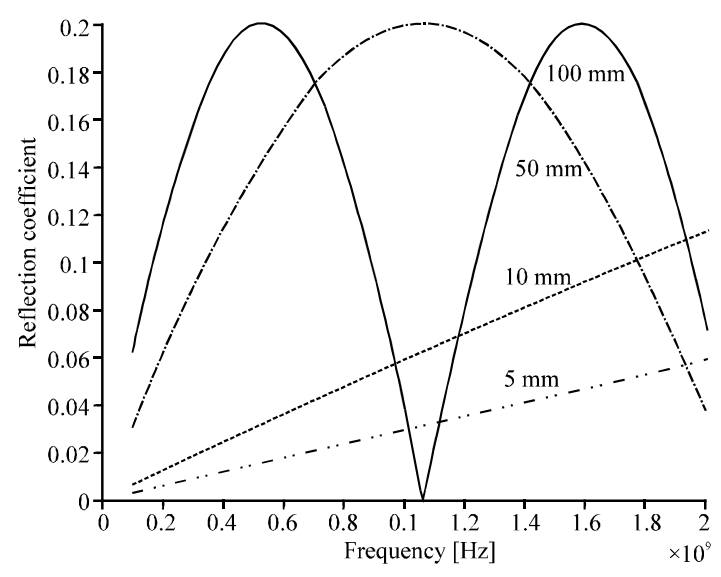

Fig. 2: Amplitude of the reflection coefficient in the frequency domain due to a thin ice-crust layer (electrical permittivity $\varepsilon=3$ ) embedded in a softer snow $(\varepsilon=2)$; the thickness of the thin layer are indicated on the plot.

The Fig. 2 plots the trend of the absolute value of the reflection coefficient, computed according the relationship $^{[5]}$, for a thin layer of an ice crust $(\varepsilon=3)$ embedded in a softer snow, with a permittivity value of $\varepsilon=2$. The general trend of the reflection versus frequency is affected by the thickness of the layer, in the selected frequency band a linear approximation yields for very thin layers $(t=5-10 \mathrm{~mm})$. For higher thickness the reflection coefficient assumes a sinusoidal behaviour with peaks at different frequencies: for a thin layer of $50 \mathrm{~mm}$ the peak is well centred on the frequency of about $1 \mathrm{GHz}$. Two peaks are present when a thin layer of $100 \mathrm{~mm}$ in thickness is considered, at the reference frequency of about $900 \mathrm{MHz}-1 \mathrm{GHz}$, the reflection coefficient is almost null, that reveals that at some particular frequencies (related to $1 / 4-1 / 2 \ldots$ of the wavelength) the reflection of layer with thickness of $100 \mathrm{~mm}$ could be negligible. As far as the amplitude of the reflection coefficient is concerned, it can be seen that at the frequency of $1 \mathrm{GHz}$ the values increase from 0.25 for a thin layer of $5 \mathrm{~mm}$, to 0.05 for the layer of 10 $\mathrm{mm}$ and to 0.2 for the layer of $50 \mathrm{~mm}$.

\section{MATERIALS AND METHODS}

The mechanical properties of snowpack are usually inferred using conventional approach to estimate the density, the cohesion and the free (liquid) water content, for instance static penetrometers are adopted to estimate the consistency by measurements of the depth to which a standard needle penetrates into the snow pack. Radar survey for snow thickness detection were conducted since 30 years ago, in North-Europe radar track-mounted systems were employed for snow thickness evaluation and for estimating the equivalent water content of snow pack $^{[12]}$ for hydrology purposes and the recharge of shallow aquifers. Experiments for detecting human body buried by avalanches were carried out in ' 80 and '90, with ambiguous results because of the practical limitations due to the slowness of the radar survey on rugged areas.

Single reflection mode with zero offset or constant offset is the conventional approach for snow pack layering and thickness estimation, it is reasonable to assess that only an estimation of the average (on the snow pack) density and water equivalent content can be carried out, as pointed out in the experiment described in the following paragraph.

A vertical cross-section of the snow cover is obtained by moving the transmitter/receiver device along a transect. The radar image displays the amplitude of the reflected wave versus the two way travel-time and the distance along the surface. Two-way traveltimes can be converted to depth if wave propagation velocity is known (for instance from direct snow thickness measures using static penetrometer device). However only in a medium with uniform density the two-way traveltime can be converted to depth assigning one average velocity without introducing significant errors in depth estimation. Otherwise the uniform density and constant water content is seldom detected in the field, especially in layered snow pack where thermo-mechanical processes provides for snow transformations, the assumption may cause relevant inaccuracies. 
Am. J. Applied Sci., 6 (3): 414-423, 2009

Multifold radar (CMP or CDP) survey provides for a better vertical resolution and deeper investigation than single reflection approach, but it is much more cost and time expensive. Therefore, on hill-slopes the multifold investigation is rarely used for mapping the snow cover on wide areas: preferentially is adopted for estimating the vertical velocity profile in some selected areas.

The spatial resolution is mainly associated to the radar wavelength and to the stratigraphic snow pack conditions, CMP or CDP data acquisition using multifold approach can provide information on density and water content through analysis of interval velocity between two reflection interfaces.

The vertical resolution of multi-fold approach is related to the presence and separation between horizontal interfaces and their delectability, the wave velocity derived from this approach refers to an average value computed according to the thickness of each horizon. The method is suitable to estimate the lateral variability of density at different depth more than offering high resolution in vertical discrimination. Using WARR approach (Wide Angle refraction and Reflection) it is possible to determine both the ground wave velocity both the interval velocity due to the reflection at the snow pack interfaces.

The data quality must be assessed taking into account the experimental inaccuracies such as the antenna positional error, the uncertainty in time picking, the time shift, the disturbance of the radar signal due to snow surface roughness and subsurface features (snow impurities and rock debris) close to the antenna.

The radiation pattern and the frenels radius determine the cross-sectional area of the interfaces in the snowpack that intercept and reflect the radiated power, in Fig. 3 the first fresnel zone radius is plotted versus the two-way traveltime for a reference wave velocity of $0.2 \mathrm{~m} \mathrm{~ns}^{-1}$ at the frequency of $1 \mathrm{GHz}$. It can be seen that at the traveltime of $10 \mathrm{~ns}$, approximately at the depth of $1 \mathrm{~m}$, the radius is equal to $0.3 \mathrm{~m}$, at the depth of 2 meters (twt $=20 \mathrm{~ns}$ ) the fresnel radius increases up to $0.45 \mathrm{~m}$. These values can be kept in mind when the lateral resolution of the radar performance would be addressed or when the effect of the snowpack-bedrock morphology on the radar response have to be considered in the estimate of the snow cover thickness.

Georadar experiments: The experiments were conducted in the Sestriere sky-domain on the Italian Alps in the Olympic Valley on the hill-slopes near to Sestriere and Sauze d'Oulx, the surveys concerned radar investigation and comparison with the

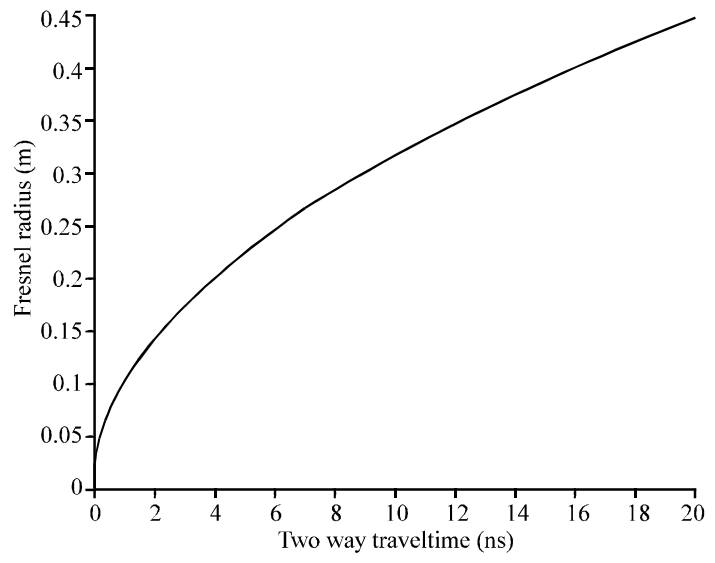

Fig. 3: First fresnel zone radius versus the two-way traveltime for a wave velocity of $0.2 \mathrm{~m} \mathrm{~ns}^{-1}$ at the frequency of $1 \mathrm{GHz}$

stratigraphic evidences coming from the snow vertical profiles of the mechanical properties of the snow covers.

Several tests were performed in different area at middle elevation (between 2000 and $2.500 \mathrm{~m}$ on the sea level) during the winter period (February-March), therefore they refer to the deposition and transformation of the fresh snow during the winter. In the area the snow falls are frequents, starting from November, the snow reaches the maximum values of 4-5 meter in thickness pack at a elevation of $2.500 \mathrm{~m}$ on the sea level. The metamorphism of the snow is usually intense due to the climatic changes, the winds modify significantly the snow conditions and provide for strong mechanical discontinuities. These climatic conditions determine different type of snow transformation during the winter and the formation of thin crusts of ice that in particular slope condition can be responsible of avalanche risk.

The conventional approach for monitoring the mechanical properties of snow pack is based on the excavation of trenches or pit (2-3 meters in depth or more according to the maximum thickness of the snow cover) where the free water content, the metamorphism of the snow crystals, the density are measured at different depth. Static penetration tests are usually performed to estimate the snow compaction and cohesion.

The GPR surveys were performed using different antennas in the frequencies band ranges between 500 $\mathrm{MHz}$ up to $1500 \mathrm{MHz}$, data were acquired both in single fold and multifold configuration. The results of surveys performed in three different sites (Vallonas, Broussailles and Fraiteve) are discussed. 
The data processing of the single fold reflection data involved:

- The marker interpolation to normalize the horizontal trace distance to a normalized trace distance of $0.125 \mathrm{~m}$

- The Time picking of the reflected signal at the snow-soil interface

- The Estimate of average wave velocity according to the data processing of CDP data

- The Depth conversion using the wave velocity detected from the CDP data

A preliminary estimate of the depth resolution considers that vertical resolution is related to the bandwidth of the received signal $\mathrm{B}$ and the shortest wavelength of the received signal bandwidth, the spectral analysis performed on the radar traces permitted to estimate an average value of wavelength of $0.24 \mathrm{~m}(500 \mathrm{MHz})$ and $0.13 \mathrm{~m}(900 \mathrm{MHz})$ : the quarterwavelength calculation yields to the theoretical vertical resolution of about $5-10 \mathrm{~cm}$.

The Vallonas test site is located on a perfectly planar soil in an well isolated small valley, where the snow accumulation is not affected by wind or human activity. Therefore the metamorphism is mainly representative of the temperature changes during the winter. The average thickness of the snow pack $(1.40-/+0.05 \mathrm{~m})$ was measured with a graduate rod steel at different points along the profiles surveyed by the GPR.

The measurements of free water content and density performed on the trench pointed out a very low free water content, this condition permitted to estimate the average density of the snow pack starting from the traveltime radar measurement and applying the relationship ${ }^{[8]}$. The single fold profiles permitted to estimate the two-way traveltimes of the substratum reflection with an accuracy of $0.5 \mathrm{~ns}$ (Fig. 4 and 5): The observed average velocity value of $0.235 \mathrm{~m} \mathrm{~ns}^{-1}$ corresponds to a snow pack density (average) of $340 \mathrm{~kg} \mathrm{~m}^{-3}$.

The weak reflection observed at the depth of about $0.7 \mathrm{~m}$ from the surface is due to the increase of the snow density from a value of $340-410 \mathrm{~kg} \mathrm{~m}^{-3}$, as estimated by the measures performed on the pit excavated in the snow.

The radar data acquired at the Broussailles site are plotted in Fig. 6 and 7, the images show two radar vertical sections of the snow pack acquired in single reflection mode, on the there are the raw data: filtered and processed image are at the bottom of the image. In the raw data the air-snow interface provides for an

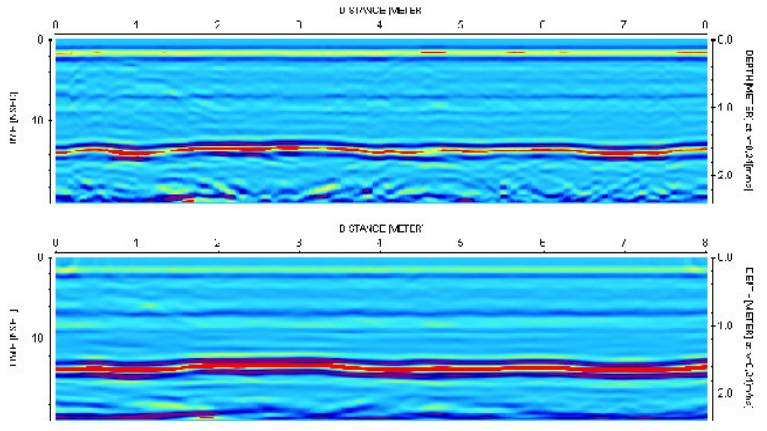

Fig. 4: Site Vallonas-Profile 1-top) raw radar data collected using a central frequency of $900 \mathrm{MHz}$, bottom) filtered and migrated image, the main reflection at 13-14 ns refers to the snow-terrain interface, the reflection 1 is related to the presence of a crust winds, snow layering is also well detected

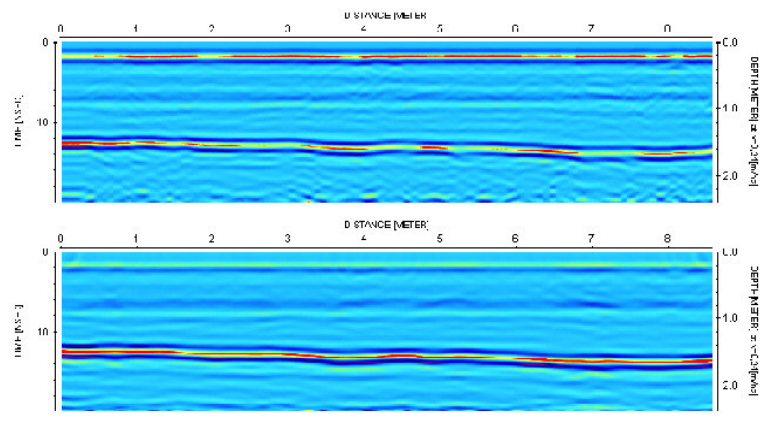

Fig. 5: Site Vallonas-Profile 2-top) raw radar data collected using a central frequency of $900 \mathrm{MHz}$; bottom) filtered and migrated image, the main reflection at 13-14 ns refers to the snow-terrain interface, the crust winds (reflections 1) appears less continuous than the image of previous figure, snow layering below reflection 1 is also well detected

intense reflection signal, the contrast of the electrical properties between the snow pack and the soil surface determines a strong reflection signal, weaker reflections, related to the snow layering, can be detected inside the snow pack.

The snow cover is characterised by three main macro-layers, according to the measures performed with a dynamometer on samples collected at different depth in a snow pit. The snow density increases from the surface to the bottom of the snow pack, according to a different compaction and metamorphic transformation, density values ranges between 290 and $425 \mathrm{~kg} \mathrm{~m}^{-3}$. 


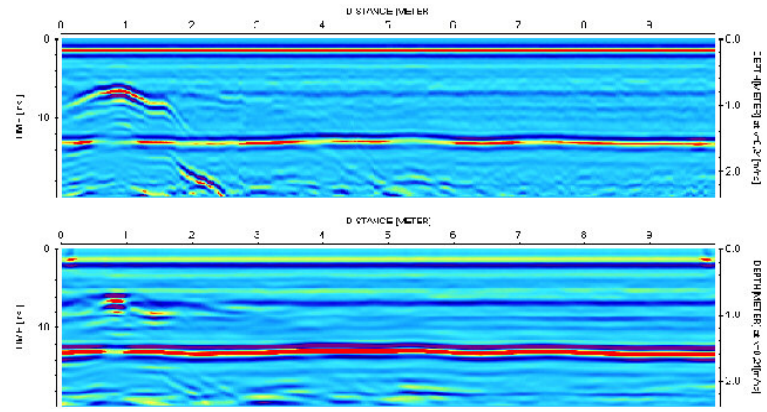

Fig. 6: Site Broussailles-Profile 1-top) raw radar data collected using a central frequency of $900 \mathrm{MHz}$, bottom) filtered and migrated image, on the left side of the radargrams is detected the reflection due to a target, two skies buried at 0.65 meters in depth, infixed on the snow from the lateral trench
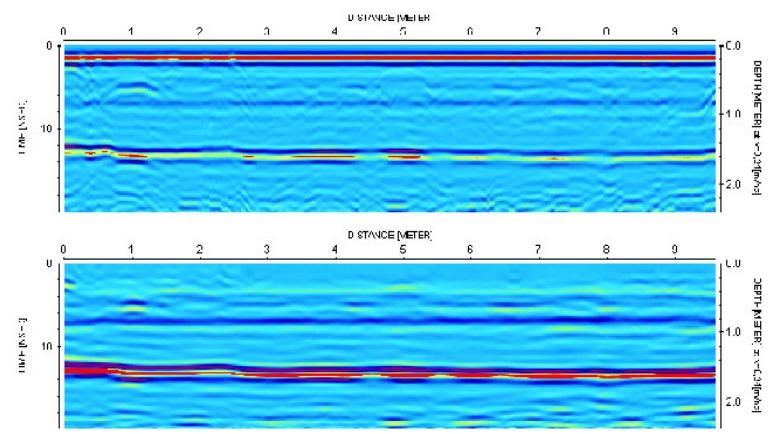

Fig. 7: Site Broussailles-Profile 2-top) raw radar data collected using a central frequency of $900 \mathrm{MHz}$, bottom) filtered and migrated image

The velocity analysis performed on CDP radar data permitted to estimate the electromagnetic wave velocity in the range between 0.23 and $0.24 \mathrm{~m} \mathrm{~ns}^{-1}$, that correspond to the electrical permittivity ranges between 1.5-1.7.

The uncertain in the density evaluation can be assessed starting from the accuracy in the electromagnetic wave velocity determined by the CDP data, an average values of $0.235+/-0.005 \mathrm{~m} \mathrm{~ns}^{-1}$ yields to an estimate of the permittivity value of $1.63-/+0.08$, according to the Looyenga model, the equivalent density values derived from radar data are in the range between 310 and $380 \mathrm{~kg} \mathrm{~m}^{-3}$. The discrepancy between the density inferred by gravimetric measures (with a dynamometer) and ones estimated by the radar data can be assigned to the inaccuracy in determining the average density value of the whole snow pack, without the possibility to estimate the density of each layer from

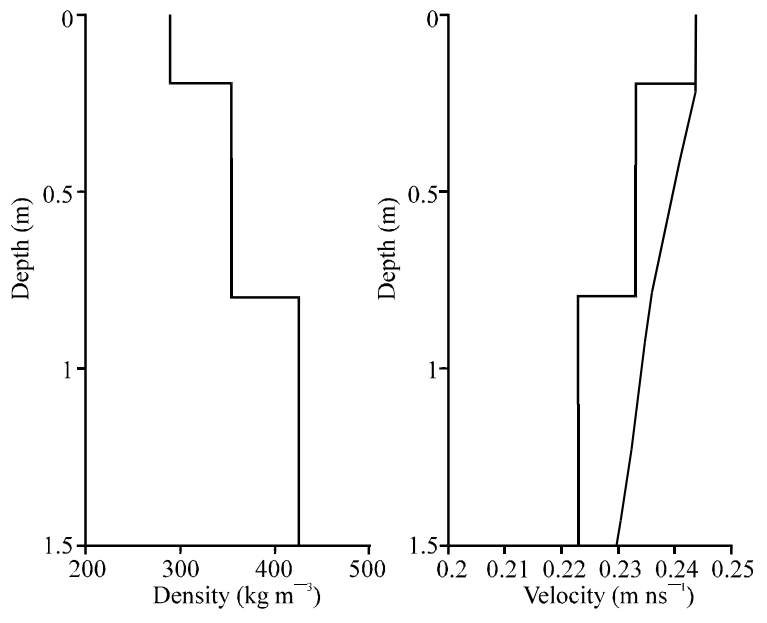

Fig. 8: Example of CDP acquisition for interval velocity or root mean square velocity estimate of the snowpack

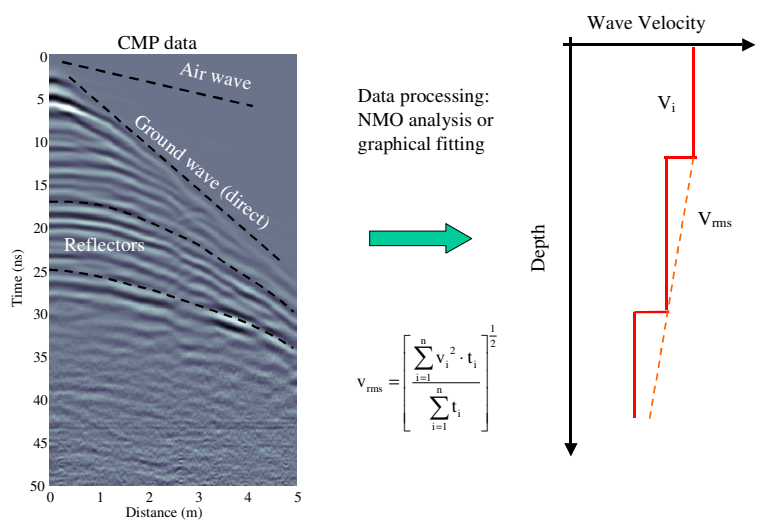

Fig. 9: Site C-Fraiteve-Profile 1-top) radar image acquired at $900 \mathrm{MHz}$, bottom) image of the envelope of instantaneous amplitude for enhancing the weak reflection due to snow layering

radar data, moreover the sensitivity of the relationship between density and electrical permittivity is rather high in the selected range and provides for an increase in the uncertainty of the density estimation, as discussed later in the next paragraph.

The Fraiteve site is located at $2550 \mathrm{~m}$ above the sea level, the area is interested by strong winds that are responsible for a significant snow transport and accumulation. The site usually presents a higher thickness of snow with respect to the rest of the slope and the formations of anomalous crust winds above low consolidate snow pack. The slope morphology and the local climatic factors are responsible for a high 


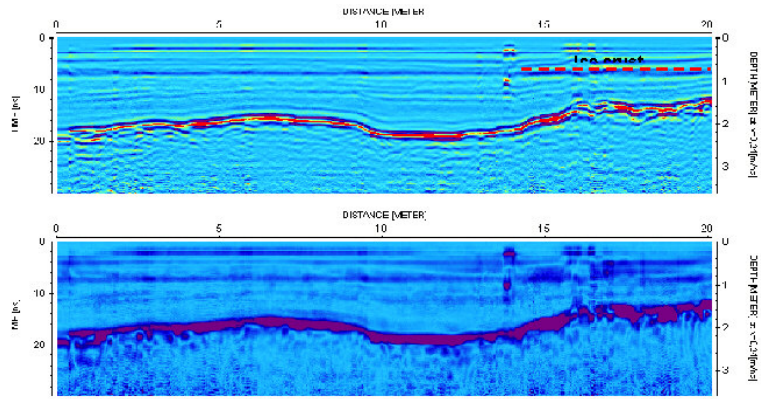

Fig. 10: Site C-Fraiteve-Profile 2-top) radar image acquired at $900 \mathrm{MHz}$, bottom) image of the envelope of instantaneous amplitude for enhancing the weak reflections due to snow layering

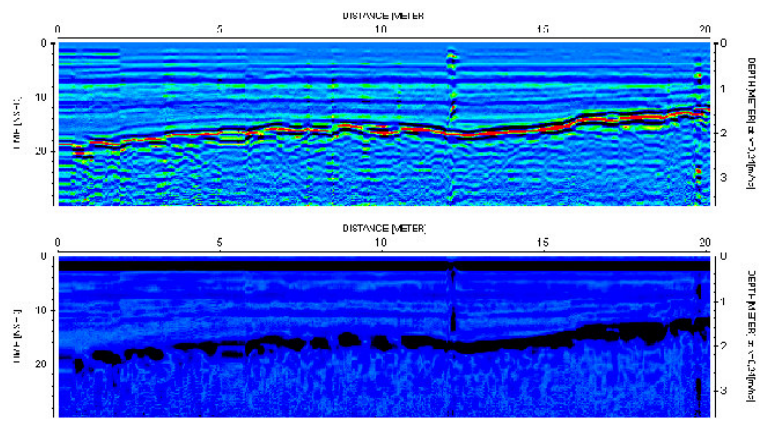

Fig. 11: Sensitivity of the Looyenga and Robin model in the density estimate starting from the error in the estimate of electrical permittivity

avalanche risk. The radar survey was performed at 500$\mathrm{MHz}$ and 900-MHz in single fold (Fig. 10 and 11) and CDP acquisition for the wave velocity estimate.

The main aim of the GPR survey was double: a fast estimate of the snow pack thickness on the top of the slope was required and the presence and the continuity of ice-crusts must be detected. The effectiveness of the radar survey was verified along two profiles by means of mechanical measurements of the snow thickness using a calibrated rod, a maximum thickness of the snow cover of about 2.5 meters was detected. The relationship ${ }^{[8]}$ was applied for determining the average value of density of the snow cover. A trench permitted to estimate the stratigraphic sequence of the snow cover, the presence of ice crusts were detected at depth of $88-90 \mathrm{~cm}$ and $112-115 \mathrm{~cm}$ from the snow surface. These crusts separates low consolidated snow layer characterised by low density, with reduced free water content and slight metamorphic transformation.

\section{RESULTS AND DISCUSSION}

The snow pack in the test sites was mainly characterised by dry-snow with very low free water content, this makes difficult to extend the results to more general snow conditions, where melting process could provide for an increase of the free water content. In such cases the estimate of density values from electromagnetic velocity analysis could be ambiguous, because the lack in reliability of the density-permittivity model here adopted. However, the radar survey permitted a fast estimate of the overall thickness of snow-cover, a qualitative analysis of the different phases of deposition (snowfalls or wind transport). This are clearly identifies specially in the single fold images acquired in the Fraiteve test site, where the older layers (closer to the permafrost) reproduce with good fidelity the morphology of the substratum, as can be observed by the analysis of the reflection events related to the snow layering.

More in detail in the test sites, the experimental results pointed out:

- A good correspondence between the radargrams and the snow pack thickness, a good lateral resolution in determining irregular snow deposition can be achieved using the $900 \mathrm{MHz}$ antenna, in fresh snow or in snow with low water content the penetration depth of the radar survey is excellent: with high frequency antenna the snow pack of up to 4 meters in depth can be analysed

- Unfortunately the high velocity of dry snow and therefore the high wavelength does not permit a satisfactory vertical resolution for accurate detection of different layers

- The discrepancy between the mechanical evaluation of density and the results of radar survey is mainly due to heterogeneity of the snow pack, inaccuracy of the mechanical measures, local terrain irregularities and average density assumption for snow pack evaluation

- The evidence of weak reflections within the snow pack are related to ice-crusts, whose presence was confirmed by the analysis of the stratigraphy along the trench

- The radargrams show the seasonal deposition and compaction of different snow layers, the first snowfalls and wind accumulation assumes a trend very similar to the terrain morphology, later accumulation are characterized by the radar reflection with a trend similar to the snow-air interface, typically when the snow coverage assumes relevant thickness 


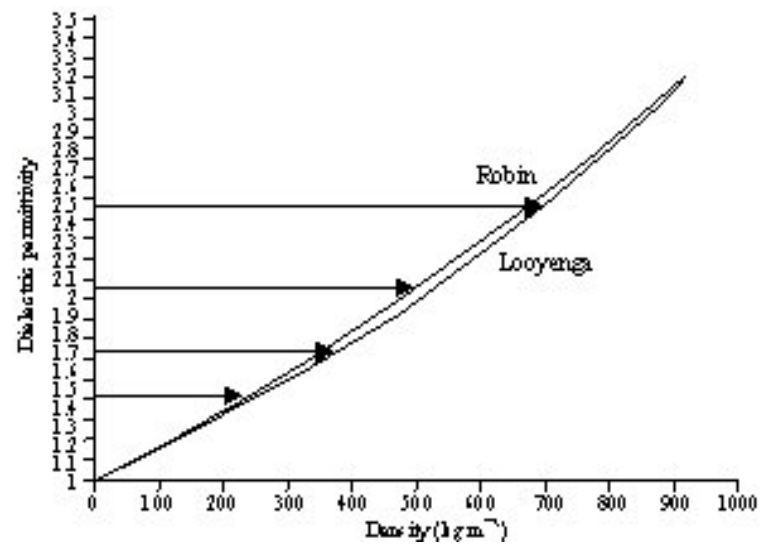

Fig. 12: The ranges of variation of the density values

The estimate of the error propagation considers the average wave velocity inferred by the CDP measurements, for instance for an average wave velocity equal to $\mathrm{v}=0.2+/-0.01 \mathrm{~m} \mathrm{~ns}^{-1}$, the error propagation on the estimate of the electrical permittivity is:

$$
\varepsilon_{\mathrm{r}}^{\prime}=\frac{\mathrm{c}^{2}}{(\mathrm{v} \pm \Delta \mathrm{v})^{2}}=\frac{0.3^{2}}{(0.2 \pm 0.01)^{2}}=\left\{\begin{array}{l}
2.04 \\
2.49
\end{array}\right\}
$$

where, $\mathrm{c}$ is the electromagnetic wave velocity in vacuum $\left(0.3 \mathrm{~m} \mathrm{~ns}^{-1}\right)$, for an average wave velocity equal to $0.24+/-0.01 \mathrm{~m} \mathrm{~ns}^{-1}$ :

$$
\varepsilon_{\mathrm{r}}^{\prime}=\frac{\mathrm{c}^{2}}{(\mathrm{v} \pm \Delta \mathrm{v})^{2}}=\frac{0.3^{2}}{(0.24 \pm 0.01)^{2}}=\left\{\begin{array}{l}
1.44 \\
1.70
\end{array}\right\}
$$

The error propagation in the density estimation is computed for the Looyenga model and Robin's equation, as documented in the Fig. 12, the plot shows the ranges of variation of the density values starting from the error in the estimate of the electrical permittivity.

It can be seen that for the average velocity of $0.2 \mathrm{~m} \mathrm{~ns}^{-1}$ the density values are in the range between 500 and $700 \mathrm{~kg} \mathrm{~m}^{-3}$, with a uncertain of about $16 \%$, at the average velocity of $0.24 \mathrm{~m} \mathrm{~ns}^{-1}$ the density is estimated in the range between 220 and $380 \mathrm{~kg} \mathrm{~m}^{-3}$, with an uncertain of about $26 \%$.

The joint application of radar survey (single fold mode) and mechanical measurements of snow pack thickness allows one to estimate the average values of density with accuracy of $5-10 \%$ for snow pack in the range of 2-3 meters. Favourable condition of snow layering, for instance alternances of ice-crusts and low consolidated snow layers, could permit the detection of the presence and spatial coherence of the ice layers.

Multifold data acquisition and velocity analysis could provide an improvement in determining the vertical density profile, a more accurate detection of snow layering and estimate of the changes of mechanical properties of the layers can be achieved using higher frequency $(>2 \mathrm{GHz})$, that in dry snow can be adopted.

\section{CONCLUSION}

The main results showed that the average value of density can be achieved with acceptable accuracy in dry snow, on the other hand the method cannot resolve the problematic task of the micro-structure of the snow.

The radar survey in single reflection mode, jointly with mechanical measurements of snow pack thickness, allows one to estimate the average values of density of the snow pack with accuracy of 5-10\% up to 2-3 meters of the snow pack thickness. Qualitatively the method, could permit the detection of the presence and spatial coherence of the ice layers or more in general to assess the snow layering inside the snow pack and the detection of alternances of ice crusts and low consolidated snow layers. The velocity analysis with the CDP approach can be successfully adopted to a fast estimate of the trend of the vertical density profile.

Several relationships can be adopted to convert the electromagnetic parameters to the density or the water equivalent snow parameters, the mixing formulas can be considered for dry snows at low density conditions, the wet snow exhibits a distinct Debye relaxation spectrum in the radiowave-microwave range and more realistic physical mixing models for small values of the free water content are required ${ }^{[13]}$.

In the selected examples, the low water content (below 3\%) of the snow permitted to adopt simplified mixing formulas with satisfactory results. However, much more efforts should be performed in order to determine more robust and accurate relationships between electromagnetic parameters and snow microstructure. For instance, imaginary part of the dielectric permittivity and frequency dispersion could be related to structure of wet snow.

\section{REFERENCES}

1. Conway, H. and J. Abrahamson, 1984. Snow stability index. J. Glaciol., 30: 321-327.

2. Annan, A.P., S.W. Cosway and T. Sigurdsson, 1994. GPR for snow water content. In: 5th International Conference on GPR. Waterloo Centre for Groundwater Research, University of Waterloo, Waterloo, Ontario, Canada, pp: 465-475. 
3. Harper, J.T. and J.H. Bradford, 2003. Snow stratigraphy over a uniform depositional surface: Spatial variability and measurement tools. Cold Regions Sci. Technol., 37: 289-298.

4. Stacheder, M., C. Huebner, S. Schlaeger and A. Brandelik, 2005. Combined TDR and LowFrequency Permittivity Measurements for Continuous Snow Wetness and Snow Density Determination. In: Electromagnetic Aquametry, Kupfer, K. (Ed.). Springer-Verlag Berlin Heidelberg, pp: 367-382.

5. Stähli, M., M. Stacheder, D. Gustafsson, S. Schlaeger, M. Schneebeli and A. Brandelik, 2004. A new in situ sensor for large-scale snow cover monitoring. Ann. Glaciol., 38: 273-278.

6. Marshall, H.P., M. Schneebeli and G. Koh, 2007. Snow stratigraphy measurements with highfrequency FMCW radar: Comparison with snow micro-penetrometer. Cold Regions Sci. Technol., 47: 108-117.
7. Kopp, 1962. Conductivitè èlectrique de la neige. Math. Phys., 13: 431-441.

8. Robin, G. and Q. de, 1975. Velocity of radio waves in ice by means of borehole interferometric techniques. J. Glaciol., 15: 151-160.

9. Birchak, J.R., C.G. Gardner, J.E. Hipp and J.M. Victor, 1974. High dielectric constant microwave probes for sensing soil moisture. Proc. IEEE, 62: 93-98.

10. Glen and Paren, 1975. The electrical properties of snow and ice. J. Glaciol., 15: 15-38.

11. Looyenga, H., 1965. Dielectric constants of heterogeneous mixtures. Phys., 31: 401-406.

12. Ulriksen, P., 1982. Application of impulse radar to civil engineering. Dept. of Eng. Geol., Univ. of Technology, Lund Sweden, pp: 175.

13. Mätzler, C., 1987. Applications of the interaction of microwaves with the seasonal snow cover. Remote Sensing Rev., 2: 259-387. 